\title{
MAP: a mnemonic for mapping BCTs to three routes to behaviour change
}

Diane Dixon ${ }^{* 1}$ and Marie Johnston ${ }^{2}$

1 University of Aberdeen

2 University of Aberdeen

${ }^{*}$ Corresponding author information: Diane Dixon, Aberdeen Health Psychology Group, Institute of Applied Health Sciences, School of Medicine and Dentistry, University of Aberdeen, Health Sciences Building, Foresterhill, Aberdeen, Scotland AB25 2ZD (E-Mail: diane.dixon@abdn.ac.uk. Telephone:+44 (0)1224 438076)

\section{Abstract: \\ Objective}

Over 90 behaviour change techniques (BCTs) have been specified but there is limited guidance to assist non-specialist practitioners in the choice of which BCTs to select for use with clients. This paper describes the development of MAP, a theory based mnemonic designed to aid practitioners in their use of BCTs. Each BCT is MAPed to one or more of three recognised routes to behaviour change, namely, Motivation development, Action control, and Prompted or cued route.

\section{Design}

A cross-sectional online discriminant content validity (DCV) questionnaire.

\section{Methods}

Fourteen judges participated, decided whether each BCT affects behaviour via each of the three routes, and provided a confidence rating for each judgement. Wilcoxon one-sample tests classified each BCT to a route or combination of routes. Intraclass correlation coefficients (ICC) assessed agreement between judges.

\section{Results}


Fifty-eight BCTs were judged to affect behaviour via a single route; 28,21 and nine $B C T s$ were judged to act via the Motivation, Action or Prompted routes respectively. Judges did not agree on a route for 35 BCTs. Overall ICC (0.89) value was high and did not differ between routes.

\section{Conclusions}

There was good agreement on candidate BCTs for interventions designed to operate through Motivation, Action or Prompted/Cued psychological processes. MAP is a mnemonic that can be used by non-specialist practitioners who implement behaviour change with their clients. MAP is not a replacement for sophisticated theory-based organisation of BCTs required for theory testing. Whilst providing practical guidance, further work is necessary to establish effectiveness of BCTs tailored to each route.

\section{Keywords:}

Behaviour change techniques, BCTs, discriminant content validity, stage models, motivation, action, prompts and cues

\section{Data availability statement:}

The data that support the findings of this study are available from the corresponding author upon request.

\section{Acknowledgements:}

The authors would like to extend their thanks to all who participated in the study. Completion of the study was a lengthy process and the time that was freely given by participants is very much appreciated. The authors also thank Tim Warren for all his support during and after their secondment to the Health Directorates. This work was partly funded by the British Psychological Society and the Scottish Government in the form of a funded secondment of the authors into the Health Directorates of the Scottish Government 
MAP: a mnemonic for mapping BCTs to three routes to behaviour change

Behaviour change is important in many contexts, including in health and health care. Evidence of the importance of behavioural factors suggests that changes in the behaviour of healthy individuals may reduce their risk of disability, morbidity and early mortality (WHO, 2011). Changes in the behaviour of people with a health condition may improve the effectiveness of treatment and their quality of life and changes in the behaviour of healthcare professionals and policy makers may lead to more effective, less risky and more efficient health services. Several systematic approaches to intervention development have been described but many are designed for use in situations where there is time, skills and resources for intervention development (O'Cathain et al., 2019). As a result these methods may not be helpful a) where behaviour is not the primary issue and therefore does not command resources, b) where there may be little time for intervention development, or c) where the practitioner is from a profession where behaviour change expertise is not a required skill, for example when a specialist asthma nurse has a consultation with a patient who has difficulty in adhering to medication. In such cases, the practitioner may rely on intuitive methods of changing behaviour and our aim is to provide them with a simple mnemonic to enable them to benefit from behavioural science.

Many methods of changing behaviour have been identified and as a result, choosing the optimal method in a specific context can prove difficult. Following recent developments in the specification of behaviour change techniques (BCTs) (Michie et al., 2013), researchers and practitioners wishing to design a health behaviour change intervention $(\mathrm{BCl})$ are faced with a choice from a taxonomy of 93 possible techniques.

There is an urgent need to link BCTs to the psychological processes theorised to effect behaviour change to enable practitioners to implement a $\mathrm{BCl}$ making best use of theory. This knowledge would be useful to aid choice of BCTs, assessment of behaviour change needs and evaluation of effects of 
behaviour change interventions. Recent work has identified links made between BCTs and 26 theoretical processes which might function as the mechanism through which the BCTs act to change behaviour. Of the 26 processes, 14 were described in the theoretical domains framework and the remaining 12 were the most frequently occurring of 83 processes identified in a systematic review of 83 behaviour change theories, which did not overlap with the processes described in the theoretical domains framework (Cane, O’Connor, \& Michie, 2012; S. Michie, West, Campbell, Brown, \& Gainforth, 2014). Evidence from literature synthesis and expert consensus methods showed agreement that, of 1456 links investigated, 92 links between BCTs and 26 theoretical processes were plausible routes to behaviour change (Carey et al., 2019; Connell et al., 2018), Johnston et al., 2020). However, the theoretical processes investigated were not connected to higher levels of theorising in behaviour change and, at a practical level, this is still a large and rather unwieldy number of links.

The challenge that we address in this paper is how to facilitate the choice of BCTs with a theoretical perspective in a practical situation where behaviour change may not be the primary challenge, and/or where there is a lack of time and/or ability to make choices between 93 BCTs or 26 theoretical approaches. Examples of such practical challenges are presented in the first column of Table 1.

This mnemonic is intended not as a replacement for more comprehensive, sophisticated theorybased organisation of behaviour change methods as would be required for theory development or testing, but for use by non-specialist practitioners in face-to-face settings with clients or by other non-behavioural scientists aiming to implement behaviour change. One might argue that they should either not engage in behaviour change, or that they should be fully trained. While additional training might be desirable, it is frequently not possible, for example if the practitioner also has continuous professional development in the more biomedical and clinical aspects of their work as in the case of the example of the specialist asthma nurse. On the other hand, it would be impossible, and perhaps irresponsible, to discourage the nurse from trying to change the behaviour of the non- 
adherent patient. Practitioners who are not trained in behavioural science may be unaware of using behaviour change methods and see it as an intuitive form of encouragement. Indeed, even when behaviour change interventions are developed and evaluated by non-behavioural scientists, the interventions may be reported without reporting any specific behaviour change methods (Scott et al, in submission). Thus our aim is to offer a mnemonic which is usable at the point at which behaviour change is required and, while requiring little behavioural science at the time of delivery, is based on guidance that is informed by current best theory and evidence. It is not a replacement for programmatic intervention development work by behavioural scientists based on full engagement with theory and the evidence base for the effectiveness of methods. Methods such as Intervention Mapping (Bartholomew, Parcel \& Kok, 1998), the Behaviour Change Wheel (Michie, van Stralen \& West, 2011) and the PRECEDE-PROCEED model (Green \& Kreuter, 1991) are designed for situations where there is time for considered development of an intervention which will be delivered in a standardised format to populations or clinical groups, a very different situation from that faced by the practitioner working with a client where the outcome of care is assessed, not as the change in behaviour, but as the change in clinical condition.

In this paper, we propose a simple route MAP via which BCTs affect behaviour. The route MAP links three theorised and empirically demonstrated routes to behaviour change to individual BCTs. The MAP can be used to identify BCTs for inclusion in interventions that are consistent with the theoretical framework of the $\mathrm{BCl}$. The MAP is intended as a useful, practical mnemonic for practitioners planning to implement behaviour change. It is not intended as a method of advancing theoretical development or of replacing more elaborated but resource-intensive methods of intervention development (O'Cathain et al., 2019).

Table 1 about here 


\section{Theoretical Background}

Dual processing models propose two main routes to behaviour change. Strack and Deutsch (2004) identify these routes as a reflective, deliberative pathway and an automatic, associative pathway (Strack \& Deutsch, 2004). They propose that change is achieved in the associative pathway by various methods, but mainly those methods involve prompts (reminders of the required behaviour) or cues (signals that elicit the behaviour).

Processes of behaviour change in the reflective pathway are outlined in social cognition models such as the Health Action Process Approach (HAPA) (Schwarzer, 1992), the Precaution Adoption Process Model (PAPM) (Weinstein, 1988); the Transtheoretical Model (TTM) (Prochaska \& DiClemente, 1984) Social Cognitive Theory (Bandura, 1997) or the Theory of Planned Behaviour (TPB) (Ajzen, 1991). Such social cognition models adopt one of two conceptualisations of behaviour change. Models such as SCT and the TPB adopt a continuous model of change. In contrast, several other models, including the HAPA, PAPM, and the TTM conceptualise behaviour change as occurring in a series of qualitatively different stages. Whilst the individual stage models differ in the number of stages proposed (the HAPA has 2 stages, the PAPM has 7 and the TTM has 6) there is a developing consensus that reflective behaviour change can be described as a two phase process; a preintentional phase during which an intention, decision, choice or goal is formed after which the individual is in a post-intentional action regulation phase (Schüz, Sniehotta, Mallach, Wiedemann, \& Schwarzer, 2009). Indeed, all three stage models have in common a pre-intentional phase and a post-intentional phase. The descriptions of each phase may differ between models but the fundamental distinction between intention development (motivation) and action regulation is inherent in all three approaches. Interventions to change behaviour will likely benefit from the inclusion of techniques that target both reflective and associative routes and both pre- and postintentional reflective processes. 
Weinstein originally proposed that the techniques necessary to change behaviour should be tailored to the different at pre- and post- intentional phases (Weinstein, 1988). It is over 20 years since Weinstein and colleagues found that persuasive messages to enhance motivation were effective for pre-intentional but not post-intentional individuals while interventions facilitating action regulation were more effective post-intentionally (Weinstein, Lyon, Sandman, \& Cuite, 1998). Reviewing the subsequent literature surrounding the most frequently used (and perhaps most complex) stage model Armitage concluded that only a small minority of the TTM literature has examined the impact of stage-matching and the evidence from those studies is mixed (Armitage, 2009). However, tailored interventions have been found to be more effective in changing behaviour in a variety of settings using different modes of delivery, varying timing of delivery and for various behaviours (Krebs, Prochaska, \& Rossi, 2010; Lustria et al., 2013; Noar, Benac, \& Harris, 2007). For example, in a systematic review of smoking cessation trials tailored interventions were more effective (Black, Eisma et al., 2020).

Thus, both theoretically and empirically there is merit in selecting BCTs to match the route(s) to behaviour change as proposed by the theoretical framework of a $\mathrm{BCl}$. Interventions that aim to exploit the reflective route will include techniques to increase motivation as well as techniques that facilitate the translation of motivation into action (Weinstein et al., 1998). Interventions that aim to exploit the associative pathway will employ techniques that prompt or cue behaviour. The main difference between prompts and cues is that prompts remind the person of things they may already have intended or been instructed to do while cues may elicit a behaviour within the person's repertoire, but without prior intention or readiness. However, both share the feature of occurring in the context in which the behaviour is enacted.

\section{MAP of three routes to behaviour change}

Based on Strack \& Deutsch's dual processing model and the accumulating agreement supporting Weinstein's separation of motivation and action, we characterise these three routes as follows. First, 
the Motivation route: (reflective, pre-intentional) behaviour change is achieved by techniques that increase conscious desire, intention or decision to change behaviour. Second, the Action regulation route: (reflective, post-intentional) behaviour change is achieved by techniques that enable the individual to act on their motivation. Third, the Prompts \& Cues route: (associative) behaviour change is achieved by techniques that do not require conscious motivation or thought being necessary at the time of acting. While the three routes are theoretically distinct, it is still possible that a BCT might act by multiple routes. For example, reviewing behavioural goals might increase both motivation and self-regulation.

These three routes (see Table 2 for detailed descriptions of each route) constitute the MAP of routes to behaviour change. In developing $\mathrm{BCls}$, on some occasions it will be appropriate to target all three routes, while in other contexts it may be appropriate to target only one route. For example, for a newly identified hazard it might be valuable to target $P$ but not $A$, while for a well-recognised hazard such as smoking it is less likely that $M$ will be effective. In Table 1 , second column, we suggest MAP routes that might be relevant for different behaviour change challenges.

However, in either case it would be valuable to have some guidance about which BCTs might be effective for each of the three MAP routes. We therefore designed an expert judgement study using the method of Discriminant Content Validation (DCV) (Johnston et al., 2014) in order to identify BCTs likely to be effective in changing behaviour via each route. These methods assess the validity of each of the three routes as a means by which a specific BCT may achieve behaviour change. They allow that a BCT may be uniquely associated with one route or judged to be effective by more than one route. Evaluating each BCT against each route enables the identification of BCTs that affect behaviour through a single $\mathrm{M}, \mathrm{A}$ or $\mathrm{P}$ route, or by multiple routes.

Three features of the DCV method are of relevance here. One, the decision task is explicit, in that it employs agreed or explicitly stated definitions. Two, it employs agreement between judges acting independently rather than by consensus. Three, it identifies, in this case, route(s) by which a BCT 
affects behaviour and route(s) by which a BCT does NOT affect behaviour. This is an especially important feature for work in applied settings because it provides information that can be used to avoid waste of resources by enabling the use of BCTs that act via route(s) relevant to a particular situation and by not delivering BCTs that do no not effect change by that route(s).

\section{Methods}

Design

An online study using DCV methods for experts to judge the validity of each of the MAP routes as a theoretical process of change behaviour for each BCT.

\section{Participants}

14 expert judges participated. Judges were all familiar with the BCTTV1 taxonomy of BCTs (Behaviour Change Technique Taxonomy Version 1) (Michie et al., 2013) and all had experience of delivering behaviour change interventions. Ten were tenured academic health psychologists, 1 a researcher and two were researcher/practitioners. Participants were recruited through personal invitation of health psychologists working in the UK, and North America.

\section{Materials}

Descriptions of the motivation, action regulation and prompts and cues routes were provided (as in Table 2). The BCT label, definition and examples of each of the 93 BCTs in the BCTTV1 were also provided as described in Michie et al, 2013.

\section{Table 2 about here}

\section{Procedure}

Participants received study information, consent details and the questionnaire via a link to the study site, hosted on the Qualtrics online research platform. Completion of the questionnaire was used to infer consent to participate. 
The descriptions of the three routes to behaviour were provided at the top of each page of the questionnaire (see Figure 1 for an example of a completed judgement of the BCT self-monitoring of the outcome(s) of behaviour). The 93 BCT labels, their definitions and examples of each were listed below the definitions. Participants were asked to decide whether each BCT affects behaviour change through each of the three routes to behaviour, and to provide a confidence rating $(0 \%-100 \%$ confident in $10 \%$ increments) for each judgement.

Figure 1 about here

\section{Analyses}

All analysis were conducted using IBM SPSS-25.

\section{Reliability of DCV judgements}

Intraclass correlation coefficient (ICC) was used to assess reliability. The ICC estimates were calculated based on a mean rating, consistency-agreement, 2-way mixed random effects model. It has been demonstrated that ICC estimates of high precision can be obtained with 12 judges (Saito, Sozu, Hamada, \& Yoshimura, 2006).

\section{Scoring of DCV data}

Confidence ratings assigned to BCTs judged to affect behaviour or not affect behaviour via a route description were divided by 100 (and multiplied by - 1 if the judge indicated the BCT does not match the route). This resulted in a scale between -1 (very confident BCT does not operate via the route) and +1 (very confident the BCT does operate via the route). Two-tailed Wilcoxon one sample tests were applied to the data against the criterion value of zero. Effect sizes were calculated by dividing the absolute (positive) standardised test statistic by the square root of the number of pairs. Benjamini-Hochberg correction was used to correct for multiple tests using $\mathrm{P} \leq 0.05$ as the starting value (Benjamini \& Hochberg, 1995). 
Statistically significant values were taken as an index that the BCT could (or could not) effect behaviour change via the specific MAP route.

Each BCT was thus classified as effecting behaviour change for each of the three MAP routes. It was possible for a BCT to be classified to one or more routes resulting in seven possible combinations of routes: $M, A, P, M A, M P, A P$, or MAP. Further, because DCV enables a 'no match' judgement, each BCT could also be classified as NOT affecting behaviour via any of the seven possible combinations of routes.

\section{Ethics}

The study was approved by the Ethics Committee of the School of Psychological Sciences and Health at the University of XX.

\section{Results}

\section{Reliability of participant performance}

ICC estimates across all judgements for all 14 judges was 0.89 . However, two judges only completed approximately half of the task, which reduced the number of judgements included in the ICC estimate to 51 from 279 judgements in total. The ICC was therefore recalculated using the data from the 12 judges who completed the task; ICC $=0.92$.

Agreement between judges was consistent across the three routes. Using data from the 12 judges who completed the task, the ICC for judgements of BCTs ascribed to the motivation, action regulation or prompts and cues routes were $0.97,0.93$ and 0.95 respectively.

Thus satisfactory agreement was found between judges and this was the case for each of the three routes.

\section{Classification of BCTs}


The number of BCTs judged to affect or not affect behaviour by each of the seven combinations of routes is shown in Table 3. There was agreement that 58 BCTs affected behaviour by at least one particular route. Twenty eight and $21 \mathrm{BCTs}$ were judged to affect behaviour via the motivation or action regulation routes respectively. Only nine BCTs were judged to affect behaviour via the prompted or cued route. No BCT was judged to affect behaviour via more than one route.

There was also agreement that 62 BCTs did not affect behaviour via a particular route. Of these, 28 $\mathrm{BCT}$ s were judged to not operate via the P route, whereas only nine and $11 \mathrm{BCTs}$ were classified as not operating via the motivation and action regulation routes.

Forty six BCTs had agreement on the route(s) via which they both did and did not affect behaviour. Twelve BCTs were classified only to the route via which they affect behaviour and 16 BCTs were classified only to the route(s) via which they did not affect behaviour. No agreement was reached on 19 BCTs.

Table 3 about here

BCTTV1 describes 93 BCTs clustered into 16 groups. The allocation of BCTs in each of the 16 groups is shown in Table 4. Eleven groups were judged to contain BCTs that affected a single route only. BCTs in eight groups were assigned to the motivation route only; namely the Natural Consequences, Comparison of Behaviour, Comparison of Outcomes, Reward and Threat, Identity, Scheduled Consequences, Self-belief and Covert Learning groups. BCTs in two groups (Goals and Planning and Social Support groups) were judged to affect behaviour via the action regulation route only. BCTs in the Associations group were judged to act via the prompted/cued route only. Five groups were judged to contain BCTs that act via more than one route. Three groups contain both motivation and action regulation BCTs (Feedback and Monitoring, Shaping Knowledge and Regulation groups) and two groups contain both action regulation and prompts/cue BCTs (Repetition and Substitution and Antecedents groups) (the labels and definitions for all 93 BCTs in BCTTV1 are provided in the Supplementary File Table 2). 
Table 4 about here

The BCTs with the top ten largest effects sizes for each route are shown in Table 5 (the classification data for each BCT, including the full Wilcoxon analyses are provided in the Supplementary File Table 1).

Table 5 about here

These strong motivation route BCTs focus on shaping understanding, through information provision and thinking about the future consequences that likely accrue from changing (or not) behaviour. In contrast, action regulation BCTs focus on 'doing' as a two stage process. Goals are set and then a series of BCTs are available to support goal directed behaviour through self-monitoring, engaging in graded tasks, behavioural practice and problem solving through difficulties. Other BCTs support selfregulation via regulation of emotion, conservation of mental resources and pharmacological and social support. Whilst, restructuring the environment is used by the BCTs judged to operate via the $\mathrm{P}$ route as well as BCTs that promote associative learning and habit formation.

\section{Discussion}

This paper describes a route MAP to behaviour change for the non-specialist, with the MAP label to act as a mnemonic. The MAP is composed of three routes to behaviour change that accommodate the two theorised pathways to behaviour, namely the associative and reflective paths (Strack \& Deutsch, 2004). The reflective path contains two routes, namely motivation development and action regulation, whereas the associative path is described by a single prompted or cued route. The DCV task identified BCTs that were reliably judged to operate via each of the three routes to behaviour and Table 5 could be used to suggest BCTs that might be used for each route. Finding that the majority of BCT groupings from BCTTv1 were judged to operate via a single route adds weight to the original groupings by further confirming agreement about the processes by which the BCTs operate. The MAP and its associated BCTs were designed to support the use of psychological theory and the developments within behaviour change science, i.e. the BCTTV1, in applied settings. We anticipate practitioners planning to implement behaviour change but with limited behaviour change training 
will use the MAP to ensure that a) they consider different interventions for clients with different problems in changing behaviour rather than 'one-size-fits-all', b) that they consider different routes or processes by which behaviour may be changed and c) they have a basis for selecting a BCT. MAP is not designed to advance theory, but to enhance the practical use of a simple theoretical framework for selecting BCTs for behaviour change interventions. In addition, where resources, skills, time and priorities permit, more sophisticated intervention development methods such as intervention mapping or the behaviour change wheel should be used ( $O^{\prime}$ Cathain et al., 2019). MAP is not designed as a replacement for these methods but to complement them in situations where these more complex methods are not applicable. Nevertheless, it would be of interest to apply MAP to other taxonomies or approaches to behaviour change or to theoretical constructs.

The MAP has several strengths. First, it provides a practical tool for practitioners and researchers when designing $\mathrm{BCl}$ s. It enables the selection of $\mathrm{BCT}$ s that operate via the route(s) to change judged to be of relevance to a given behaviour in a given context. Additionally, it offers guidance on BCTs which might not be effective. Alternatively, if the route to change is unclear MAP enables BCTs to be selected that affect each of the three routes, thereby ensuring that both the reflective and associative pathways are engaged to promote change. For example, returning to the challenges in Table 1, the second column proposes the route the $\mathrm{BCl}$ might target based on existing evidence. The third column suggests BCTs that might be used to target that route based on the higher scoring BCTs listed in Table 4. Finally, the fourth column proposes how these BCTs might be fashioned into a $\mathrm{BCl}$. It is the case that many, or perhaps most, practitioners tasked with changing the behaviour of their clients or patients are not experts in behaviour change theory. MAP will be of particular value to such practitioners because it provides a useful mnemonic, based on high level theorising, to support their delivery of BCTs that address each or all of the three routes to behaviour.

This practical utility of MAP has been already been demonstrated. MAP is the basis of a national training programme to enable NHS health and social care staff to support people to change and 
maintain their behaviour to improve their health and wellbeing (NES, 2018). The MAP training programme includes training to deliver BCTs that operate via each of the three routes and a system for deciding which BCTs to use and when: for example, when faced with a highly motivated client the practitioner would know to include BCTs that target the A route and, perhaps the P route where appropriate. In addition, a MAP based national training programme for health professionals involved in diabetes care has trained over 200 GPs, specialist nurses, podiatrists, physiotherapists and consultants across Scotland and has shown positive outcomes in intention to utilise BCTs in clinical practice, and successful use of planning techniques (BLINDED; Swanson \& Maltinsky, 2019). It is possible that the value of MAP to practitioners can serve as a basis for more enhanced approaches, or for higher levels of training being provided routinely for practitioners whose clinical work frequently involves behaviour change.

Second, it provides a basis for identifying and analysing the psychological processes involved in effective BCls. Each route is affected by multiple BCTs and each BCT, for which there was agreement, was judged to affect behaviour via a single route. Together, this understanding enables evidence synthesis based on psychological processes, in addition to the usual synthesis based on components of $\mathrm{BCls}$, such as $\mathrm{BCTs}$, mode of delivery etc. Work to understand common processes via which disparate interventions effect change has previously led to step changes in understanding, for example self-efficacy was originally proposed as the common pathway for the effectiveness of quite different interventions for phobia (Bandura, 1977).

Third, although the MAP may appear simple, it is able to support a broad diversity of approaches to behaviour change. The MAP is not only applicable to individual level $\mathrm{BCls}$ delivered by health professionals it can also be used to inform higher level interventions for behaviour change. For example, the associative pathway, represented by the P-route and its BCTs would accommodate legislative interventions and interventions based on nudge technology. In addition, it also supports, via $B C T$ s that operate via environmental change, social models of behaviour, consequently, MAP can 
be used by a variety of academic disciplines and to support interdisciplinary working required for complex interventions.

That said, the BCT-route links are currently based on expert judgement alone and this is accompanied by some obvious limitations. Fewer BCTs were reliably judged to affect the P route, as compared with the $\mathrm{M}$ or $\mathrm{A}$ routes. This lack of agreement likely reflects the less frequent use of the $\mathrm{P}$ route in $\mathrm{BCls}$ perhaps because the reflective pathway is predominant in the social cognition models typically used in the development of BCls (Bull et al., 2018; Michie, Abraham, Whittington, McAteer, \& Gupta, 2009). Similarly, practitioners are also likely to be more familiar with BCTs, such as goal setting, self-monitoring and action planning, than $\mathrm{P}$ route BCTs. This combination of more frequent theorising with and greater practical use of reflective route BCTs would promote greater consistency of and confidence in the judgements reported herein.

Further, the proposed links between BCTs and routes to change is based on expert opinion and we would caution against regarding them as definitive or conclusive as expert opinion is not a substitute for empirical evidence. Some empirical support for the MAP routes is provided in a meta-analysis of smoking cessation trials which found that BCTs addressing self-regulatory and associative processes, i.e. the action regulation and prompts and cues routes, were more effective than those directed at reflective motivational processes suggesting support for the differentiating routes (Black, Johnston et al.,2020). There is a need for empirical investigation to establish the processes via which BCTs effect behaviour change and the MAP provides a structure that is practical for this purpose compared with other, more complex frameworks. Other recent work identified 1456 links between BCTs and 26 theoretical processes, 92 of which were judged to be plausible routes to behaviour change using a process of triangulation between literature review and expert consensus (Carey et al., 2019; Connell et al., 2018; Johnston et al., 2020). This level of complexity will be difficult to investigate empirically even for the most skilled researchers and will likely severely restrict its use by practitioners. The simple route MAP described here is based on higher level theorising in behaviour 
change that identifies two pathways to behaviour, composed of three routes to change. This

simplicity will support detailed empirical testing of the proposed links between the three routes in

MAP and the BCTs described in BCTTV1 and other intervention components that aim to change

behaviour.

\section{References}

Ajzen, I. (1991). The Theory of Planned Behavior. Organizational Behavior and Human Decision Processes, 50(2), 179-211.

Armitage, C. J. (2009). Is there utility in the transtheoretical model? British Journal of Health Psychology, 14(2), 195-210. doi:10.1348/135910708X368991

Bandura, A. (1977). Self-efficacy: Toward a unifying theory of behavioural change. Psychological Review, 84(2), 191-215. doi: 10.1037/0033-295X.84.2.191

Bandura, A. (1997). Self-Efficacy: The Exercise of Control. New York: W. H.Freeman.

Bartholomew, L. K., Parcel, G. S., \& Kok, G. (1998). Intervention Mapping: A process for developing theory- and evidence based health education programs. Health Education and Behavior, 25(5), 545-563. DOI: 10.1177/109019819802500502

Benjamini, Y., \& Hochberg, Y. (1995). Controlling the false discovery rate - a practical and powerful approach to multiple testing. Journal of the Royal Statistical Society Series B-Methodological, 57(1), 289-300. doi.org/10.1111/j.2517-6161.1995.tb02031.x

Black, N., Eisma, M. C., Viechtbauer, W., Johnston, M., West, R., Hartmann-Boyce, J., . . Marijn de Bruin, M. (2020). Variability and effectiveness of control group interventions in smoking cessation trials: A systematic review and meta-analysis. [published online ahead of print, 2020 Feb 11]. Addiction. 2020;10.1111/add.14969.

Black, N., Johnston, M., Michie, S., Hartmann-Boyce, J., West, R., Viechtbauer, W., ... \& de Bruin, M. (2020). Behaviour change techniques associated with smoking cessation in intervention and comparator groups of randomized controlled trials: a systematic review and metaregression. [published online ahead of print, 2020 Mar 20]. Addiction. 2020;10.1111/add.15056. doi:10.1111/add.15056

Bull, E. R., McCleary, N., Li, X., Dombrowski, S. U., Dusseldorp, E., \& Johnston, M. (2018). Interventions to promote healthy eating, physical activity and smoking in low-income groups: a systematic review with meta-analysis of behavior change techniques and delivery/context. International Journal of Behavioral Medicine, 25(6), 605-616. doi:10.1007/s12529-018-9734-z

Cane, J., O'Connor, D., \& Michie, S. (2012). Validation of the theoretical domains framework for use in behaviour change and implementation research. Implementation Science, 7(1), 37. doi: 10.1186/1748-5908-7-37

Carey, R. N., Connell, L. E., Johnston, M., Rothman, A. J., de Bruin, M., Kelly, M. P., \& Michie, S. (2019). Behavior change techniques and their mechanisms of action: a synthesis of links described in published intervention literature. Annals of Behavioral Medicine, 53(8):693-707. doi: $10.1093 / a b m /$ kay078

Conn, V. S., Ruppar, T. M., Enriquez, M., \& Cooper, P. (2016). Medication adherence interventions that target subjects with adherence problems: Systematic review and meta-analysis.

Research in Social and Administrative Pharmacy, 12(2), 218-246. doi: 10.1016/j.sapharm.2015.06.001 
Connell, L. E., Carey, R. N., de Bruin, M., Rothman, A. J., Johnston, M., Kelly, M. P., \& Michie, S. (2018). Links between behavior change techniques and mechanisms of action: an expert consensus study. Annals of Behavioral Medicine, 53(8),708-720. doi: 10.1093/abm/kay082.

Debono, D., Taylor, N., Lipworth, W., Greenfield, D., Travaglia, J., Black, D., \& Braithwaite, J. (2017). Applying the Theoretical Domains Framework to identify barriers and targeted interventions to enhance nurses' use of electronic medication management systems in two Australian hospitals. Implementation Science, 12(1), 42. DOI: 10.1186/s13012-017-0572-1

Dorsey, R., \& Songer, T. (2011). Lifestyle behaviors and physician advice for change among overweight and obese adults with prediabetes and diabetes in the United States, 2006. Preventing chronic disease, 8(6), A132-A132.

French, D. P., Olander, E. K., Chisholm, A., \& Mc Sharry, J. (2014). Which behaviour change techniques are most effective at increasing older adults' self-efficacy and physical activity behaviour? A systematic review. Annals of Behavioral Medicine, 48(2), 225-234. doi: 10.1007/s12160-014-9593-z.

Green, L.W., \& Kreuter, M.W. (1991). Health Promotion Planning: An Educational and Environmental Approach, 2nd edition. Palo Alto: Mayfield Publishing Co.

Johnston, M., Dixon, D., Hart, J., Glidewell, L., Schröder, C., \& Pollard, B. (2014). Discriminant content validity: A quantitative methodology for assessing content of theory-based measures, with illustrative applications. British Journal of Health Psychology, 19(2), 240-257. doi: 10.1111/bjhp.12095

Johnston, M., Carey, R.N., Connell Bohlen, L.E., Johnston, D.W., Rothman, A.J., de Bruin, M., Kelly, M.P., Groarke, H. \& Michie, S. (2020). Development of an online tool for linking behavior change techniques and mechanisms of action based on triangulation of findings from literature synthesis and expert consensus. Translational Behavioral Medicine

Johnston, M., Carey, R.N., Connell Bohlen, L., Johnston, D., Rothman, A., Bruin, M., . . Michie, S. (in submission). Linking behavior change techniques and mechanisms of action: Triangulation of findings from literature synthesis and expert consensus. Annals of Behavioral Medicine

Krebs, P., Prochaska, J. O., \& Rossi, J. S. (2010). A meta-analysis of computer-tailored interventions for health behavior change. Preventive Medicine, 51(3), 214-221. doi: 10.1016/j.ypmed.2010.06.004

Lustria, M. L. A., Noar, S. M., Cortese, J., Van Stee, S. K., Glueckauf, R. L., \& Lee, J. (2013). A MetaAnalysis of web-delivered tailored health behavior change interventions. Journal of Health Communication, 18(9), 1039-1069. doi: 10.1080/10810730.2013.768727

Michie, S., Abraham, C., Whittington, C., McAteer, J., \& Gupta, S. (2009). Effective techniques in healthy eating and physical activity interventions: a meta-regression. Health Psychology, 28(6), 690-701. doi: 10.1037/a0016136

Michie, S., Richardson, M., Johnston, M., Abraham, C., Francis, J., Hardeman, W., . . Wood, C. (2013). The Behavior Change Technique Taxonomy ( $v 1$ ) of 93 hierarchically clustered techniques: building an international consensus for the reporting of behavior change interventions. Annals of Behavioral Medicine, 46(1), 81-95. doi: 10.1007/s12160-013-9486-6

Michie, S., van Stralen, M.M. \& West, R. (2011). The behaviour change wheel: A new method for characterising and designing behaviour change interventions. Implementation Sci 6, 42. doi: 10.1186/1748-5908-6-42

Michie, S., West, R., Campbell, R., Brown, J., \& Gainforth, H. (2014). ABC of Behaviour Change Theories. Great Britain: Silverback Publishing.

Morrissey, E. C., Corbett, T. K., Walsh, J. C., \& Molloy, G. J. (2016). Behavior change techniques in apps for medication adherence: a content analysis. American Journal of Preventive Medicine, 50(5), e143-e146. doi: 10.1016/j.amepre.2015.09.034

NHS Education Scotland. (2018). MAP Health Behaviour Change Training Programme. NES: Edinburgh. 
Noar, S. M., Benac, C. N., \& Harris, M. S. (2007). Does tailoring matter? Meta-analytic review of tailored print health behavior change interventions. Psychological Bulletin, 133(4), 673-693. doi: 10.1037/0033-2909.133.4.673.

Noordman, J., van der Weijden, T., \& van Dulmen, S. (2012). Communication-related behavior change techniques used in face-to-face lifestyle interventions in primary care: A systematic review of the literature. Patient Education and Counseling, 89(2), 227-244. doi: 10.1016/j.pec.2012.07.006

O'Cathain, A., Croot, L., Sworn, K., Duncan, E., Rousseau, N., Turner, K., . . Hoddinott, P. (2019). Taxonomy of approaches to developing interventions to improve health: a systematic methods overview. Pilot and Feasibility Studies, 5(1), 41. doi: 10.1186/s40814-019-0425-6

Prochaska, J. O., \& DiClemente, C. C. (1984). The Transtheoretical Approach: Towards a Systematic Eclectic Framework Homewood, IL, USA: Dow Jones Irwin

Saito, Y., Sozu, T., Hamada, C., \& Yoshimura, I. (2006). Effective number of subjects and number of raters for inter-rater reliability studies. Stat Med, 25(9), 1547-1560. DOI: 10.1002/sim.2294.

Schüz, B., Sniehotta, F. F., Mallach, N., Wiedemann, A. U., \& Schwarzer, R. (2009). Predicting transitions from preintentional, intentional and actional stages of change. Health Education Research, 24(1), 64-75. doi: 10.1093/her/cym092

Schwarzer, R. (1992). Self-Efficacy in the adoption and maintenance of health behaviours: theoretical approaches and a new model. In R. Schwarzer (Ed.), Self-efficacy: Thought Control of Action (pp. 217-245). Bristol:PA: Taylor Francis.

Scott, C., deBarra, M., Johnston, M. deBruin, M., Scot, N., Matheson, C, Bond, C \& Watson, M (in submission) Using the Behaviour Change Technique Taxonomy (BCTTV1) to identify the active ingredients of pharmacist interventions to improve patient health outcomes.

Strack, F., \& Deutsch, R. (2004). Reflective and impulsive determinants of social behavior. Personality and Social Psychology Review, 8(3), 220-247. doi: 10.1207/s15327957pspr0803_1

Swanson, V., \& Maltinsky, W. (2019). Motivational and behaviour change approaches for improving diabetes management. Practical Diabetes, 36(4), 121-125. doi: 10.1002/pdi.2229

Weinstein, N. D. (1988). The precaution adoption process. Health Psychology, 7(4), 355-386. doi: 10.1037/0278-6133.7.4.355

Weinstein, N. D., Lyon, J. E., Sandman, P. M., \& Cuite, C. L. (1998). Experimental evidence for stages of health behavior change: the precaution adoption process model applied to home radon testing. Health Psychology, 17(5), 445-453. doi: 10.1037/0278-6133.17.5.445

WHO. (2011). Global health risks: mortality and burden of disease attributable to selected major risks. Retrieved from Geneva, Switzerland:

https://www.who.int/healthinfo/global burden disease/GlobalHealthRisks report full.pdf 


\section{Figure Captions}

Figure 1: Illustration of the DCV questionnaire showing a completed judgement for the BCT 'Selfmonitoring of outcome(s) of behaviour' 


\section{ROUTE DEFINITIONS}

\section{Motivation Route Techniques}

Techniques that change behaviour by increasing the person's motivation i.e. their conscious desire to do the target behaviour, by making beliefs or feelings about the behaviour more favourable (or by making beliefs and feelings about unwanted behaviours less favourable). Motivational

techniques lead toward (and may or may not reach): a behavioural goal, or an intention, or a decision, or the selection of a specific behaviour to enact.

\section{Action Regulation Route Techniques}

Techniques that change behaviour by enabling the motivated individual to self-regulate towards the goal or implement the intention, decision or selected behaviour. Self-regulation is a systematic process involving conscious efforts to modulate thoughts, emotions and behaviours in order to achieve goals within a changing environment (Zeidner, et al, 2000). It involves: setting goals, attending to and remembering goals; making plans for action to achieve goals; making plans for coping to achieve goals in the face of barriers; appraising progress and revising goals and strategies; and selfcontrol over cognitive and emotional processes. It may include the development and/or placing prompts to influence later action.

\section{Prompted or Cued Route Techniques}

Techniques that change behaviour by triggering action by associative automatic processes without requiring conscious motivation or thinking about self-regulation at the time of action. Associative processes are triggered by stimuli present at the time of action and these may be, for example: environmental, cognitive, physiological, emotional, social. Stimuli may be prompts, i.e. they trigger behaviour compatible with the individual's

motivation and goals; (Prompts may on occasion remind or draw the attention to these behavioural goals). Stimuli may be cues, i.e. stimuli that elicit behaviourindependent of motivation and goals.

\section{Self-monitoring of outcome(s) of behavior}

Establish a method for the person to monitor and record the outcome(s) of their behavior as part of a behavior change strategy

Example: Ask the person to weigh themselves at the end of each day, over a two week period, and record their daily weight on a graph to increase exercise behaviors

\begin{tabular}{|l|c|c|c|c|c|c|c|c|c|c|c|c|c|c|}
\hline & \multicolumn{10}{|c|}{$\begin{array}{l}\text { Route to } \\
\text { behaviour? }\end{array}$} & \multicolumn{8}{c|}{ How confident are you in each of your judgements? } \\
& Yes & No & $0 \%$ & $10 \%$ & $20 \%$ & $30 \%$ & $40 \%$ & $50 \%$ & $60 \%$ & $70 \%$ & $80 \%$ & $90 \%$ & $100 \%$ \\
\hline Motivation & & $\checkmark$ & & & & & & & & $\checkmark$ & & & \\
\hline Action & $\checkmark$ & & & & & & & & & & & $\checkmark$ & \\
\hline Prompts/Cues & & $\checkmark$ & & & & & & & & & & & $\checkmark$ \\
\hline
\end{tabular}


Table 1: Illustration of practical challenges with a suggested theoretical route to behaviour change and how results of the judgement task might be incorporated in a behaviour change intervention

\begin{tabular}{|c|c|c|c|}
\hline $\begin{array}{l}\text { Practical behaviour change } \\
\text { challenge }^{+}\end{array}$ & $\begin{array}{l}\text { Theoretical process: } \\
\text { Route to behaviour } \\
\text { change }\end{array}$ & $\begin{array}{l}\text { Possible } \mathrm{BCTs}^{\ddagger} \text { (BCT number from } \\
\qquad \mathrm{BCTTV1)}\end{array}$ & Possible $\mathrm{BCl}$ \\
\hline $\begin{array}{l}\text { Patient with borderline diabetes } \\
\text { who could benefit from weight } \\
\text { loss }^{\text {b }}\end{array}$ & M & $\begin{array}{l}\text { Credible source (9.1) } \\
\text { Information about health } \\
\text { consequences (5.1) } \\
\text { Incompatible beliefs (13.3) }\end{array}$ & $\begin{array}{l}\text { GP or nurse as credible sources outlines the possible } \\
\text { health consequences of increasing activity }\end{array}$ \\
\hline $\begin{array}{l}\text { Hospital managers with poor } \\
\text { reporting of adverse events }{ }^{c}\end{array}$ & $\mathrm{P}$ & $\begin{array}{l}\text { Prompts/Cues (7.1) } \\
\text { Restructuring the physical } \\
\text { environment (12.1) } \\
\text { Adding objects to the environment } \\
\text { (12.5) }\end{array}$ & $\begin{array}{l}\text { Health board produces an online reporting tool linked } \\
\text { to the hospital electronic diary system. Individual } \\
\text { managers receive a diary prompt each week at the } \\
\text { same time to complete the reporting tool }\end{array}$ \\
\hline
\end{tabular}




\begin{tabular}{|c|c|c|c|}
\hline $\begin{array}{l}\text { Stroke patient with poor } \\
\text { adherence to medication due to } \\
\text { impaired memory }\end{array}$ & $\mathrm{P}$ & $\begin{array}{l}\text { Prompts/cues (7.1) } \\
\text { Habit formation (8.3) } \\
\text { Adding objects to the environment } \\
(12.5)\end{array}$ & $\begin{array}{l}\text { Plan to place medication near toothbrush in order to } \\
\text { develop the habit of taking medication at time of } \\
\text { tooth brushing using the toothbrush as a prompt and } \\
\text { placing the medication in that specific environment }\end{array}$ \\
\hline $\begin{array}{l}\text { Sedentary older person } \\
\text { agreeing but not implementing } \\
\text { an exercise programme }\end{array}$ & $A$ & $\begin{array}{l}\text { Graded tasks ( } 8.7) \\
\text { Self-monitoring of outcome(s) of } \\
\text { behaviour (2.4) }\end{array}$ & $\begin{array}{l}\text { Identify a series of graded exercises that gradually } \\
\text { increase in challenge posed. Produce a diary } \\
\text { containing a graded exercise timetable and self- } \\
\text { monitoring of outcomes, e.g. feelings, behaviours } \\
\text { consequent from performing the target graded } \\
\text { exercise }\end{array}$ \\
\hline
\end{tabular}

${ }^{\dagger}$ these practical challenges have been addressed by: ${ }^{b}$ (Dorsey \& Songer, 2011; Noordman, van der Weijden, \& van Dulmen, 2012); '(Debono et al., 2017);

d(Conn, Ruppar, Enriquez, \& Cooper, 2016; Morrissey, Corbett, Walsh, \& Molloy, 2016); (French, Olander, Chisholm, \& Mc Sharry, 2014). ${ }^{\ddagger}$ The suggested BCTs are based on the data generated by the discriminant content validity study described herein 
Table 2: Description of each route to behaviour change (as provided to judges)

Route Description

Motivation Techniques that change behaviour by increasing the person's motivation i.e. their conscious desire to do the target behaviour, by making beliefs or feelings about the behaviour more favourable (or by making beliefs and feelings about unwanted behaviours less favourable). Motivational techniques lead toward (and may or may not reach): a behavioural goal, or an intention, or a decision, or the selection of a specific behaviour to enact.

Action Regulation Techniques that change behaviour by enabling the motivated individual to self-regulate towards the goal or implement the intention, decision or selected behaviour. Self-regulation is a systematic process involving conscious efforts to modulate thoughts, emotions and behaviours in order to achieve goals within a changing environment (Zeidner, et al, 2000). It involves: setting goals, attending to and remembering goals; making plans for action to achieve goals; making plans for coping to achieve goals in the face of barriers; appraising progress and revising goals and strategies; and self-control over cognitive and emotional processes. It may include the development and/or placing prompts to influence later action 
thinking about self-regulation at the time of action. Associative processes are triggered by stimuli present at the time of action and these may be, for example: environmental, cognitive, physiological, emotional, social. Stimuli may be prompts, i.e. they trigger behaviour compatible with the individual's motivation and goals; (Prompts may on occasion remind or draw the attention to these behavioural goals). Stimuli may be cues, i.e. stimuli that elicit behaviour independent of motivation and goals. 
Table 3: Number of BCTs judged to change and not change behaviour change by each route

\begin{tabular}{lcccccccc}
\hline & \multicolumn{7}{c}{ Route to Behaviour Change } \\
Number BCTs & M & A & P & MA & MP & AP & MAP \\
\hline Operate via this route & 28 & 21 & 9 & 0 & 0 & 0 & 0 \\
Do not operate by this route & 9 & 11 & 28 & 0 & 1 & 13 & 0 \\
\hline
\end{tabular}


Table 4: The allocation of BCTs to and not to the three routes for each BCT group as described in BCTTV1

\begin{tabular}{|c|c|c|c|c|c|c|c|}
\hline \multirow{3}{*}{$\mathrm{BCT}_{\text {grouping }}^{\dagger}$} & \multirow{3}{*}{$\begin{array}{l}\mathrm{N}^{\circ} \text { of BCTs } \\
\text { in group }\end{array}$} & \multicolumn{6}{|c|}{$\begin{array}{c}\text { Number of BCTs allocated and not } \\
\text { allocated to each route }\end{array}$} \\
\hline & & \multicolumn{3}{|c|}{ Allocated } & \multicolumn{3}{|c|}{ Not Allocated } \\
\hline & & $M$ & A & $P$ & $\mathrm{M}$ & $\mathrm{A}$ & $P$ \\
\hline 1. Goals and Planning & 9 & 0 & 6 & 0 & 2 & 0 & 7 \\
\hline 2. $\quad$ Feedback and Monitoring & 7 & 3 & 3 & 0 & 0 & 0 & 7 \\
\hline 3. Social Support & 3 & 0 & 3 & 0 & 0 & 0 & 1 \\
\hline 4. Shaping Knowledge & 4 & 1 & 1 & 0 & 0 & 0 & 4 \\
\hline 5. Natural Consequences & 6 & 5 & 0 & 0 & 0 & 5 & 5 \\
\hline 6. Comparison of Behaviour & 3 & 2 & 0 & 0 & 0 & 2 & 3 \\
\hline 7. Associations & 8 & 0 & 0 & 5 & 2 & 4 & 0 \\
\hline 8. Repetition and Substitution & 7 & 0 & 4 & 1 & 1 & 0 & 1 \\
\hline 9. Comparison of Outcomes & 3 & 3 & 0 & 0 & 0 & 3 & 3 \\
\hline 10. Reward and Threat & 11 & 5 & 0 & 0 & 0 & 2 & 1 \\
\hline 11. Regulation & 4 & 1 & 3 & 0 & 1 & 1 & 3 \\
\hline
\end{tabular}




\begin{tabular}{|l|c|c|c|c|c|c|c|}
\hline 12. Antecedents & 6 & 0 & 1 & 3 & 4 & 0 & 1 \\
\hline 13. Identity & 5 & 3 & 0 & 0 & 0 & 3 & 4 \\
\hline 14. Scheduled Consequences & 10 & 1 & 0 & 0 & 0 & 2 & 0 \\
\hline 15. Self-belief & 4 & 3 & 0 & 0 & 0 & 0 & 2 \\
\hline 16. Covert Learning & 3 & 1 & 0 & 0 & 0 & 2 & 0 \\
\hline
\end{tabular}

${ }^{+}$numbering and group label taken from BCTTv1 (Susan Michie et al., 2013). Text in BOLD indicates those groupings containing BCTs assigned to one route only. 
Table 5: The "top 10" BCTs ranked according to effect size for each route to behaviour

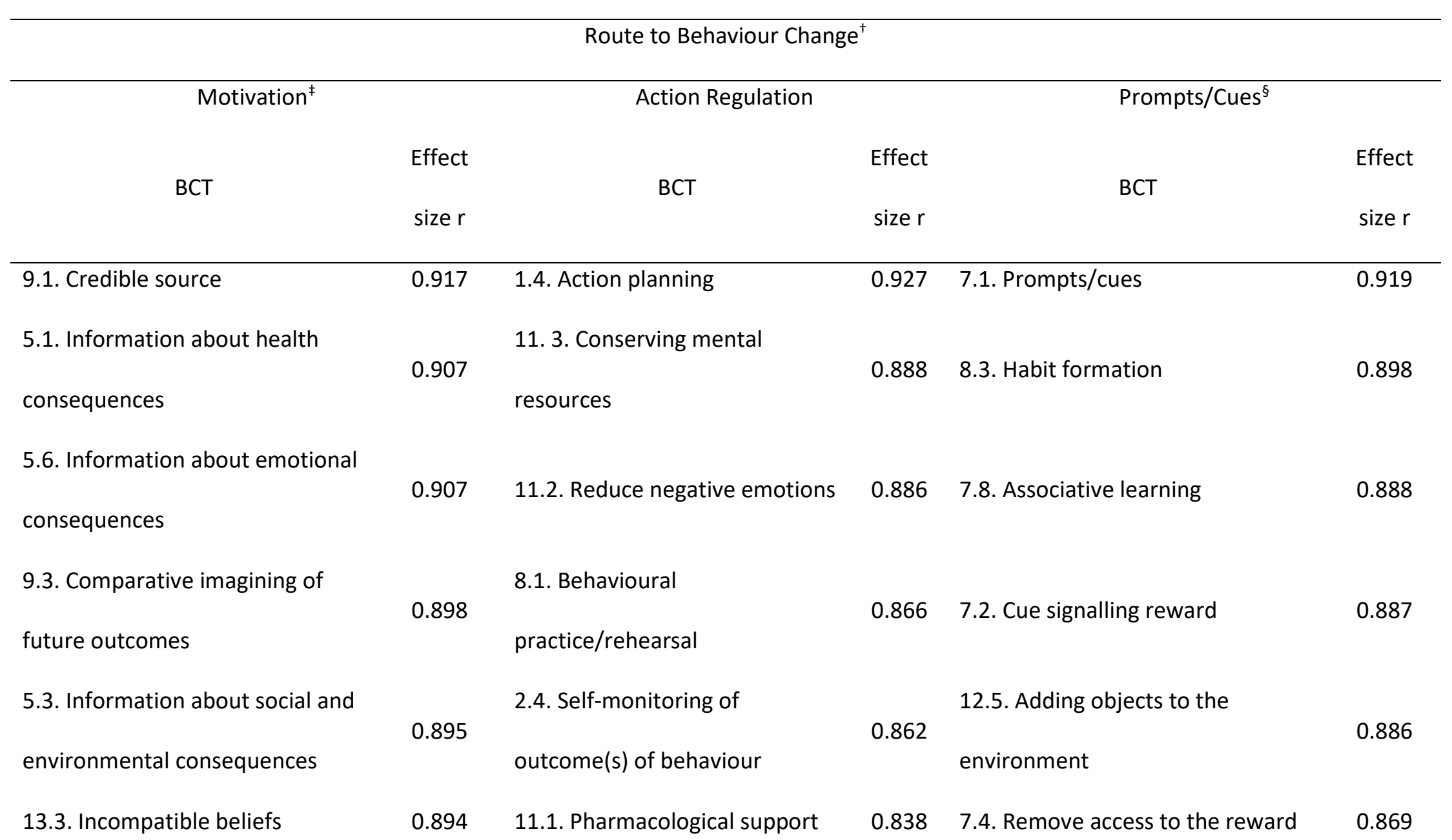


13.1. Identification of self as role

model

5.2. Salience of consequences

5.5. Anticipated regret

10.11 Future punishment

11.4. Paradoxical instructions
0.889

8.7. Graded tasks

0.889

1.2. Problem solving

0.888

1.1. Goal setting (behaviour)
12.3. Avoidance/reducing exposure

0.835

to cues for the behaviour

12.1. Restructuring the physical

0.831

environment

0.818

${ }^{\dagger}$ all data are significant at .02 level after correction for familywise error. ${ }^{\ddagger} 11 \mathrm{BCTs}$ are listed for the M route because of equal effect sizes. ${ }^{8}$ only nine BCTs achieved a significant effect size for the P route. Each BCT number and label is taken from BCTTv1 (Susan Michie et al., 2013) 